\title{
The pressure-induced local structural change around tungsten in silicate glass
}

\author{
K. Ozawa ${ }^{1 *} \oplus$, K. Hirose $^{1,2} \odot$, Y. Kuwayama ${ }^{1} \odot$, Y. Takahashi ${ }^{1,3} \oplus$
}

Abstract

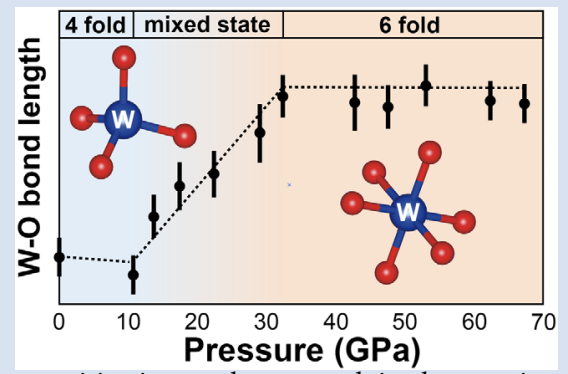

Tungsten is one of the key elements to understand the conditions and timing of planetary core formation. While the metal-silicate partitioning of tungsten has been extensively studied, the effects of pressure and silicate melt composition have been controversial. Here we have investigated the local environment of tungsten in a basaltic glass up to 67 GPa based on EXAFS spectroscopy and found that the $\mathrm{W}-\mathrm{O}$ bond length increases in a pressure range from 10 to $32 \mathrm{GPa}$, indicating that $\mathrm{W}^{6+}$ ion increases the coordination number from four to six. It is known that the coordination of silicon also changes at similar pressure range, suggesting that the coordination structure of trace element tungsten may be controlled by the $\mathrm{Si}-\mathrm{O}$ coordination. The coordination change of tungsten will affect its metal-silicate partitioning and may explain the previously observed change in the pressure effect around $5 \mathrm{GPa}$, when considering the difference between melt and glass. This also suggests further change in the pressure dependence above $32 \mathrm{GPa}$ where tungsten is predominantly sixfold coordinated. In addition, the effect of silicate melt composition may diminish at such pressure range.

Received 23 December 2020 | Accepted 2 April 2021 | Published 1 June 2021

\section{Introduction}

The Earth's mantle is depleted in siderophile (iron-loving) elements because they were preferentially partitioned into metals during core formation. Such depletion can be a record of conditions for core-forming metal segregation from silicate. Among siderophile elements, the metal-silicate partitioning of tungsten has been extensively studied because of its chemical properties and geological significance. Since tungsten is a highly refractory element, its bulk Earth abundance is obtained from the chondritic abundance without correction for volatility (see review by McDonough, 2014), giving the tungsten content in the core from the known mantle abundance. The distribution of tungsten between the core and the mantle is thought to be key to understanding the conditions for metal-silicate chemical equilibrium during core metal segregation.

Previous experiments have explored the effects of pressure, temperature, oxygen fugacity, and the compositions of metallic liquid and silicate melt on the metal-silicate partitioning of tungsten (Walter and Thibault, 1995; O'Neill et al., 2008; Cottrell et al., 2009; Siebert et al., 2011; Rai and van Westrenen, 2014; Jennings et al., 2020). However, partitioning is still not well understood even at relatively low pressures. It has been argued that tungsten becomes more siderophile with increasing pressure to $\sim 5 \mathrm{GPa}$ and then less siderophile at higher pressures (Cottrell et al., 2009; Rai and van Westrenen, 2014). This was attributed to the formation of $\mathrm{W}^{4+}$ (Cottrell et al., 2009) due to the emergence of octahedrally coordinated silicon in silicate melts (Sanloup et al., 2013). Sanloup et al. (2011) also pointed out that a change in the compressibility of liquid iron may affect the metal-silicate partitioning of trace elements including tungsten.

Pressure-induced structural changes in silicate melt and glasses have been examined, focusing on a change in the coordination of silicon (e.g., Sanloup et al., 2013; Prescher et al., 2017). The local structure around a trace element in silicate melt may be important for its partitioning, in particular for cations with high valences such as $\mathrm{W}^{6+}$, but it has been least explored. Keppler and Rubie (1993) reported the coordination changes of $\mathrm{Ni}^{2+}$ and $\mathrm{Co}^{2+}$ in silicate melts at high pressures up to $10 \mathrm{GPa}$ based on crystal field spectroscopy on quenched glasses at ambient conditions. The coordination environments of lutetium and xenon in silicate glasses and melts were examined by using in situ high pressure X-ray diffraction (XRD) techniques to $8 \mathrm{GPa}$ (de Grouchy et al., 2017; Leroy et al., 2018). As far as we know, there are no experimental studies that investigate the local structure around trace elements under lower mantle conditions so far, because of their low concentrations and disturbance by Compton signals from diamond in XRD measurements at high pressure in a diamond-anvil cell (DAC).

Here we examine a change in the coordination structure of tungsten in a basaltic glass with increasing pressure to $67 \mathrm{GPa}$, based on in situ high pressure $\mathrm{X}$-ray absorption spectroscopy in the fluorescence yield mode, which gives element selective information and is sensitive to trace elements. The extended X-ray absorption fine structure (EXAFS) and X-ray absorption near-edge

\footnotetext{
Department of Earth and Planetary Science, The University of Tokyo, Bunkyo, Tokyo 113-0033, Japan

Earth-Life Science Institute, Tokyo Institute of Technology, Meguro, Tokyo 152-8550, Japan

Photon Factory, Institute of Materials Structure Science, High Energy Accelerator Research Organization (KEK), Tsukuba, Ibaraki 305-0801, Japan

Corresponding author (email: keisuke-ozawa996@g.ecc.u-tokyo.ac.jp)
} 

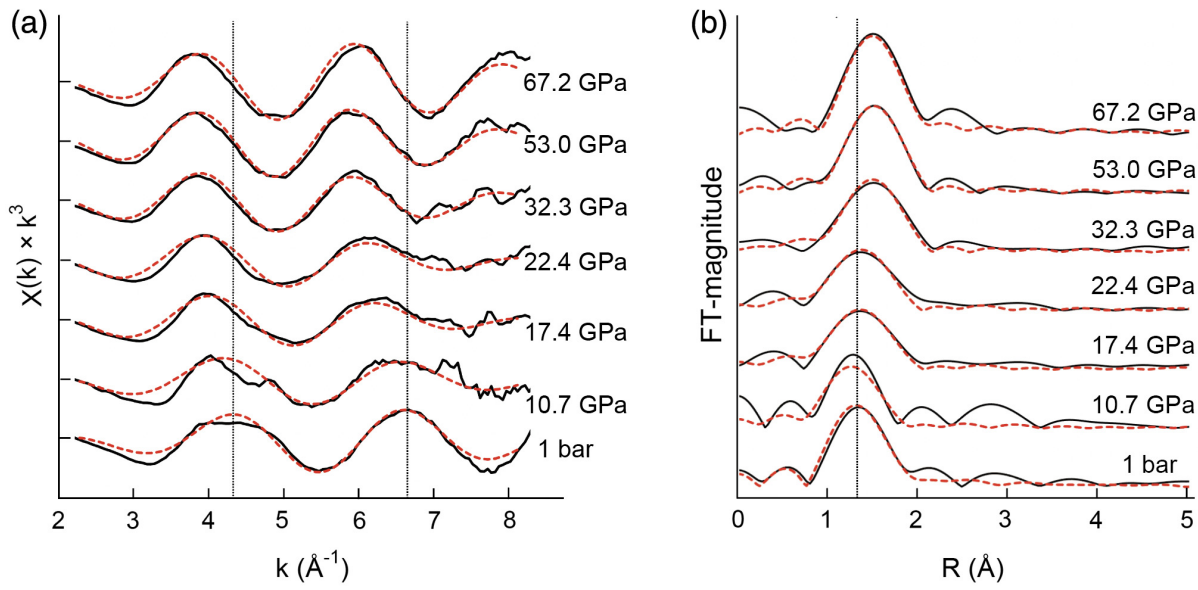

Figure 1 EXAFS oscillations of tungsten in a basaltic glass and FT-EXAFS spectra up to $67 \mathrm{GPa}$. (a) The $\mathrm{k}^{3}$-weighted oscillations, $\chi(\mathrm{k}) \times \mathrm{k}^{3}$, extracted from EXAFS spectra (black curves). The vertical dotted lines highlight the wave numbers $k$ of the first and second maxima of

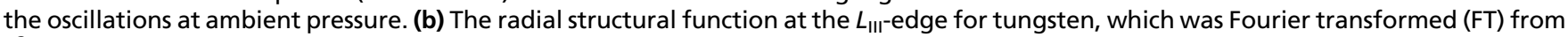
$\mathrm{k}^{3}$-weighted EXAFS oscillations (black curves). The vertical dotted line indicates the position of the maximum FT-magnitude at 1 bar. The red curves in (a) and (b) show simulation data of EXAFS spectra using the parameter extracted by FEFF.

structure (XANES) spectra demonstrate the pressure-induced coordination change of $\mathrm{W}^{6+}$. We discuss its implications for the partitioning behaviour of tungsten at high pressure.

\section{Results and Discussion}

Change in the coordination structure of tungsten. We obtained twelve separate tungsten $L_{\mathrm{III}}$-edge EXAFS spectra of the $\mathrm{W}$-doped basaltic silicate glass in a wide pressure range from 1 bar to $67.2 \mathrm{GPa}$, whose $\mathrm{k}^{3}$-weighted oscillations are shown in Figure 1a. As can be seen from the change in the oscillation period, the average $\mathrm{W}-\mathrm{O}$ bond became longer with increasing pressure from 10.7 to $32.3 \mathrm{GPa}$ although it did not change outside of this pressure range (Fig. 2). The $\mathrm{W}-\mathrm{O}$ bond length $\left(\mathrm{r}_{\mathrm{W}-\mathrm{O}}\right)$ was determined at each pressure from the EXAFS analysis (Fig. 1, Table 1)

At ambient pressure, we obtained $r_{\mathrm{W}-\mathrm{O}}=1.77 \pm 0.02 \AA$ in the basaltic glass, similar to $\mathrm{r}_{\mathrm{W}-\mathrm{O}}=1.78 \AA$ in $\mathrm{WO}_{4}{ }^{2-}$ solution reported by Kashiwabara et al. (2013). This indicates that $\mathrm{W}^{6+}$ was predominantly tetrahedrally coordinated at 1 bar in our glass sample in agreement with $\mathrm{O}^{\prime} \mathrm{Neill}$ et al. (2008). At $10.7 \mathrm{GPa}$, we found $\mathrm{r}_{\mathrm{W}-\mathrm{O}}=1.75 \pm 0.02 \AA$, which is close to the average $\mathrm{W}$-O bond length at ambient pressure. Nevertheless, the XANES spectra and their second derivatives, which are sensitive to the local structure around an element of interest, show a difference between the two pressures (Fig. 3), possibly suggesting that the $\mathrm{WO}_{4}$ tetrahedral symmetry was slightly changed by compression.

A rapid increase in the average $\mathrm{W}-\mathrm{O}$ bond length in a pressure range from 10.7 to $32.3 \mathrm{GPa}$ can be attributed to a change in the mean coordination number from four to six (Fig. 2). $\mathrm{r}_{\mathrm{W}-\mathrm{O}}=1.94 \AA$ at $32.3 \mathrm{GPa}$ is longer by $0.17 \AA$ than $\mathrm{r}_{\mathrm{W}-\mathrm{O}}=1.77 \AA$ at 1 bar. This difference in the average $\mathrm{W}$-O bond length is similar to the difference in the ionic radii between 0.42 and $0.60 \AA$ for fourfold and sixfold coordinated $W^{6+}$, respectively (Shannon, 1976). Above $32.3 \mathrm{GPa}$, the average $\mathrm{W}-\mathrm{O}$ bond length remained similar; neither the period of EXAFS oscillations nor the XANES spectra changed significantly (Figs. 1, 3). This could be because the effect of compression was compensated by the effect of the small continuous increase in the mean coordination number of $\mathrm{W}^{6+}$ to $>6$.

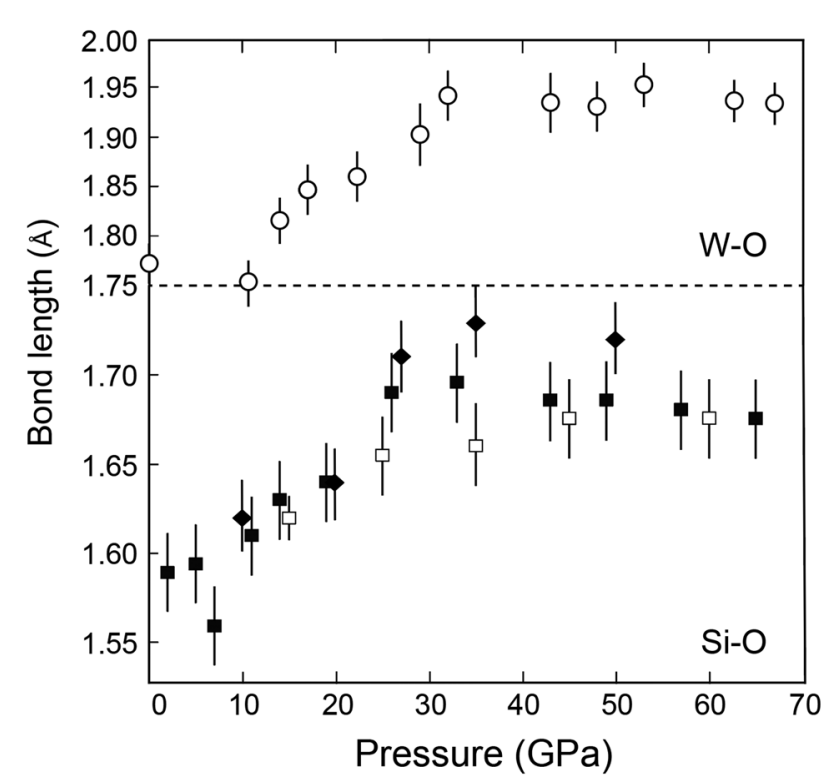

Figure 2 Pressure-induced change in the $\mathrm{W}$-O bond length (open circles) in basaltic glass on compression from the present EXAFS analyses, compared to those in the $\mathrm{Si}$-O bond length. Filled squares (Prescher et al., 2017) and diamonds (Sato and Funamori, 2010) indicate data on $\mathrm{SiO}_{2}$ glass. Open squares are for a molten basalt (Sanloup et al., 2013). All the Si-O bond length data were obtained by XRD measurements.

Coordination changes of tungsten and silicon at similar pressure range. These changes in the average $\mathrm{W}-\mathrm{O}$ bond length and the tungsten coordination number take place at a pressure range very similar to that in which silicon changes its coordination from four to six in silicate glass and melt (see a comparison in Fig. 2). Previous XRD measurements have examined the pressure effect on the average $\mathrm{Si}-\mathrm{O}$ bond length and the coordination number of silicon in $\mathrm{SiO}_{2}$ glass up to a megabar pressure (Prescher et al., 2017; Sato and Funamori, 2010). The most recent experiments performed by Prescher et al. (2017) demonstrated that i) below $10 \mathrm{GPa}$, the $\mathrm{Si}-\mathrm{O}$ bond length was constant at $\sim 1.62 \AA$, while volume was reduced because of the collapse of void space (topological change), ii) between 10 
Table 1 The $\mathrm{W}-\mathrm{O}$ bond length in a basaltic glass at each pressure obtained from the $\mathrm{k}$ range of EXAFS spectra provided. Standard deviations are given in parentheses.

\begin{tabular}{|c|c|c|}
\hline $\begin{array}{c}\text { Pressure } \\
(\mathrm{GPa})\end{array}$ & $\begin{array}{c}\text { k range } \\
\left(\AA^{-1}\right)\end{array}$ & $\begin{array}{c}\text { W-O bond } \\
\text { length }(\AA)\end{array}$ \\
\hline 0 & $2.3-8.2$ & $1.771(18)$ \\
10.7 & $2.3-8.2$ & $1.753(19)$ \\
13.7 & $2.3-8.2$ & $1.814(21)$ \\
17.4 & $2.3-8.2$ & $1.845(23)$ \\
22.4 & $2.3-8.2$ & $1.858(23)$ \\
29.0 & $2.3-7.0$ & $1.900(29)$ \\
32.3 & $2.3-8.2$ & $1.939(21)$ \\
42.7 & $2.3-7.0$ & $1.932(28)$ \\
47.5 & $2.3-8.2$ & $1.928(21)$ \\
53.0 & $2.3-8.2$ & $1.950(20)$ \\
62.3 & $2.3-8.2$ & $1.934(19)$ \\
67.2 & $2.3-8.2$ & $1.931(19)$ \\
\hline
\end{tabular}

and $40 \mathrm{GPa}$, the $\mathrm{Si}-\mathrm{O}$ bond length increased to $\sim 1.69 \AA$ as a consequence of the increase in the mean silicon coordination number from four to six, and iii) above $40 \mathrm{GPa}$, the $\mathrm{Si}-\mathrm{O}$ bond length slightly decreased with pressure, as illustrated in Figure 2. Furthermore, the XRD study on a basaltic melt by Sanloup et al. (2013) showed that the Si-O bond length rapidly increased from $<10$ to $\sim 35 \mathrm{GPa}$ (Fig. 2), consistent with the observations in $\mathrm{SiO}_{2}$ glass. The ab initio simulations of a model basalt by Bajgain et al. (2015) reported that the mean silicon coordination number started to increase from $5.3 \mathrm{GPa}$ at 2,200 K. They additionally calculated the mean coordination numbers of other major cations at high pressures, demonstrating that those of network modifier cations of $\mathrm{Na}^{+}, \mathrm{Ca}^{2+}, \mathrm{Mg}^{2+}$, and $\mathrm{Fe}^{2+}$ increase with initial compression to $20 \mathrm{GPa}$, in contrast to those of network former cations of $\mathrm{Si}^{4+}$ and $\mathrm{Al}^{3+}$.

These suggest that the coordination increase in tungsten may be induced by that of silicon which determines the overall networking structure in silicate. Below $10 \mathrm{GPa}, \mathrm{W}^{6+}$ is predominantly fourfold coordinated and therefore cannot be a network former in a silicate glass (Farges et al., 2006). This is explained by the bond valence theory; oxygen cannot connect fourfold coordinated $\mathrm{W}^{6+}$ and $\mathrm{Si}^{4+}$ ions because the sum of the valence units combining $\mathrm{W}^{6+}-\mathrm{O}(\mathrm{VI} / 4=1.5)$ and $\mathrm{Si}^{4+}{ }_{-} \mathrm{O}$ bonds $(\mathrm{IV} / 4=1.0)$ exceeds the valence $(=2)$ of an oxygen ion (Pauling, 1929). Therefore, the $\mathrm{WO}_{4}$ tetrahedra are positioned in a region that is rich in the network modifier cations (e.g., $\mathrm{Na}^{+}, \mathrm{Ca}^{2+}, \mathrm{Mg}^{2+}$, and $\mathrm{Fe}^{2+}$ ), and are disconnected from the framework of $\mathrm{SiO}_{2}$ tetrahedra. This could be the reason why tungsten is much less siderophile during metal-silicate partitioning when silicate melt exhibits higher $\mathrm{NBO} / \mathrm{T}$ (the ratio of non-bridging oxygens per tetrahedrally coordinated cations, a measure of the depolymerisation) (Walter and Thibault, 1995) or contains more alkali metals and alkaline earth metals, in particular $\mathrm{CaO}$ (Jennings et al., 2020). Above $10 \mathrm{GPa}$, fourfold coordinated $\mathrm{Si}^{4+}$ starts to change into sixfold coordination, leading to an increase in bridging oxygen at the expense of non-bridging oxygen and possibly the oxygen constituting $\mathrm{WO}_{4}$ tetrahedra. While fourfold coordinated $\mathrm{W}^{6+}$ cannot bind to the bridging oxygen due to its high bond valence, it is possible for sixfold coordinated $W^{6+}$ to do so because its bond valence units decrease to $1.0(=\mathrm{VI} / 6)$. Such sixfold coordinated $\mathrm{W}^{6+}$ can thus be a network former cation, bonding to the bridging oxygen together with the charge compensating cations such as $\mathrm{Na}^{+}$in a basaltic glass.

Implications for the partitioning of tungsten. The change in the nature of the $\mathrm{W}^{6+}-\mathrm{O}$ bond will affect the partitioning behaviour of tungsten. Cottrell et al. (2009) examined the partitioning of tungsten between liquid iron and basaltic melt and showed positive and negative pressure effects on the metal/ silicate partition coefficient below and above 2-6 GPa, respectively. A more recent study by Rai and van Westrenen (2014) also demonstrated the positive pressure effect below $5 \mathrm{GPa}$ before it changes to negative at higher pressures. Cottrell et al. (2009) argued that such a change in the pressure effect may correspond to the emergence of $\mathrm{W}^{4+}$, but this was not supported by Wade et al. (2012) who found that the valence of tungsten remained six in silicate melt at 6 to $24 \mathrm{GPa}$ based on augmented experimental data. This might be attributed to the change in the structure of liquid iron alloy (Sanloup et al., 2011; Shibazaki et al., 2015), but the pressure response of the metal-silicate partitioning of tungsten is different from that of nickel and cobalt, which cannot be explained solely by the structural change in liquid metal. Alternatively, it is likely that the change in the pressure
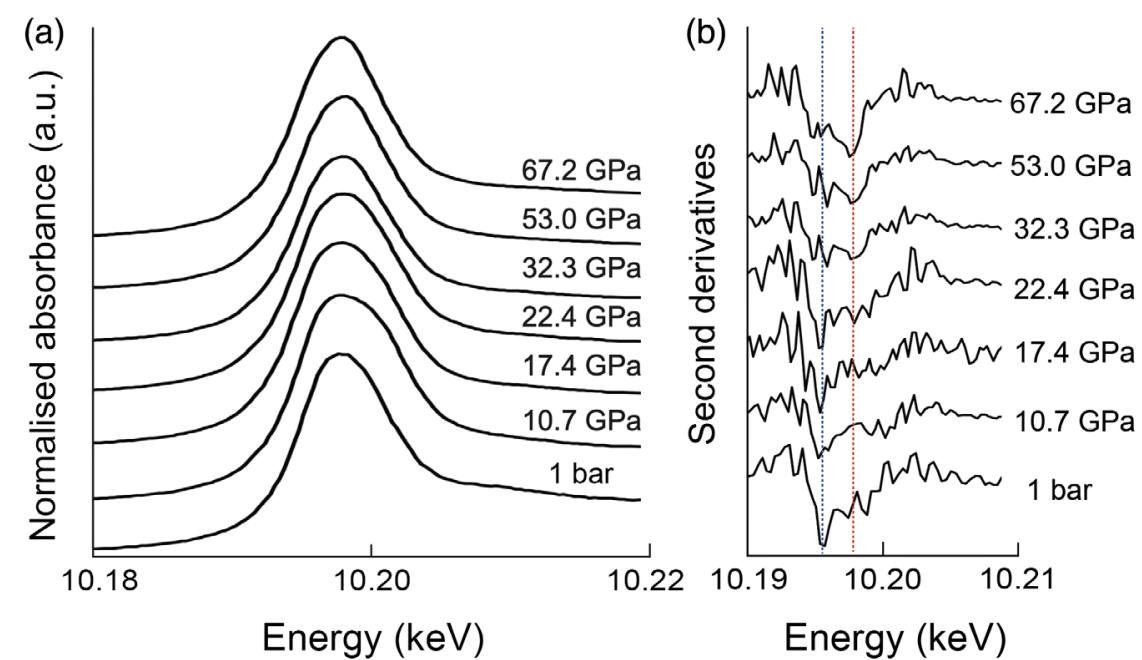

Figure 3 Tungsten $L_{11}$-edge XANES spectra of a basaltic glass collected up to $67 \mathrm{GPa}$ after background subtraction. (a) Normalised XANES spectra and (b) their second derivatives. In (b), the blue and red dashed lines indicate the position of the minimum of the second derivatives of the XANES spectrum at 1 bar and that of the local minimum that is prominent above $32.3 \mathrm{GPa}$, respectively. These XANES spectra show a clear difference between fourfold and sixfold coordinated $\mathrm{W}^{6+}$. 
effect on the metal/silicate partitioning of tungsten above $\sim 5 \mathrm{GPa}$ is caused by the onset of the increase in the coordination number of $\mathrm{W}^{6+}$ in a silicate melt (note that this may start in melt at pressures slightly lower than that in glass as predicted for Si by Bajgain et al., 2015). These observations further suggest that the pressure effect on the metal/silicate partitioning of tungsten above $32 \mathrm{GPa}$ (sixfold coordinated $\mathrm{W}^{6+}$ predominant) is likely different from that at $<5 \mathrm{GPa}$ (fourfold coordinated $\mathrm{W}^{6+}$ predominant) and 5-32 GPa (mixed state).

It has also been argued that the metal-silicate partitioning of tungsten strongly depends on the silicate melt composition represented by such as $\mathrm{NBO} / \mathrm{T}$ or the $\mathrm{CaO}$ content as mentioned above (e.g., Walter and Thibault, 1995; Jennings et al., 2020). For example, according to the parameterisation by $\mathrm{O}^{\prime} \mathrm{Neill}$ et al. (2008), the partition coefficient of tungsten between metal and pyrolitic melt is forty times as high as that between metal and basaltic melt below $6 \mathrm{GPa}$. Such strong compositional dependence may significantly diminish as the coordination structure of tungsten changes. Indeed, the earlier experiments conducted by Siebert et al. (2011) have demonstrated that the effect of NBO/T on metal-silicate partitioning is smaller for sixfold coordinated, high valence cations such as $\mathrm{Nb}^{5+}$ and $\mathrm{Ta}^{5+}$ than for fourfold coordinated $\mathrm{W}^{6+}$ and $\mathrm{P}^{5+}$. We can thus expect that the effect of silicate melt composition on the metal-silicate partitioning of tungsten is smaller above $32 \mathrm{GPa}$, where sixfold coordinated $\mathrm{W}^{6+}$ is predominant, than previously found below $10 \mathrm{GPa}$.

Moreover, the change in the coordination number of tungsten should affect its isotopic fractionation between melt and crystals at high pressure. In general, heavier isotopes tend to be partitioned into a phase with a lower coordination number (Kashiwabara et al., 2017). Therefore, the ${ }^{182} \mathrm{~W} /{ }^{184} \mathrm{~W}$ ratio in silicate melt equilibrated with metallic liquid above 32 GPa may be higher than that below $10 \mathrm{GPa}$, although the temperature for metal-silicate equilibrium at the time of core formation may have been too high to cause a resolvable isotopic fractionation, as is the case for Mo as reported by Hin et al. (2019).

Coordination changes of other trace elements. It is worth emphasising that the pressure range for the coordination change of $\mathrm{W}^{6+}$ observed in this study is quite different from those for $\mathrm{Ni}^{2+}, \mathrm{Co}^{2+}$ (Keppler and Rubie, 1993), and $\mathrm{Lu}^{3+}$ (de Grouchy et al., 2017), whose coordination numbers increase rapidly with increasing pressure up to $10 \mathrm{GPa}$. Such a difference may be related to the field strength ( $=$ charge/ionic radius) of these trace elements. The low field strength of $\mathrm{Ni}^{2+}, \mathrm{Co}^{2+}$, and $\mathrm{Lu}^{3+}$ suggests that their local structures are similar to those of network modifier cations. On the other hand, we expect that the local structures around high field strength elements such as $\mathrm{Mo}^{6+}$, $\mathrm{P}^{5+}$, and $\mathrm{As}^{5+}$ are close to that of $\mathrm{W}^{6+}$, and therefore their coordination numbers will increase in a manner similar to that of tungsten. Future studies of these high field strength elements besides tungsten will clarify the importance of the field strength on the behaviour of pressure-induced coordination changes in silicate glasses and melts.

\section{Acknowledgements}

We thank H. Sakuma for discussion on the cause of pressureinduced coordination change of tungsten. K. Ohta is acknowledged for his help in DAC experiments. Comments from two anonymous reviewers helped to improve the manuscript. This work was supported by JSPS Kakenhi grants no. 16H06285 and 20J21667. This work was performed with the approval of Photon Factory (proposal no. 2018S1-001 and 2020G670).

\section{Additional Information}

Supplementary Information accompanies this letter at https:// www.geochemicalperspectivesletters.org/article2116.

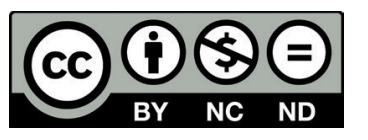

(C) 2021 The Authors. This work is distributed under the Creative Commons Attribution NonCommercial No-Derivatives 4.0 License, which permits unrestricted distribution provided the original author and source are credited. The material may not be adapted (remixed, transformed or built upon) or used for commercial purposes without written permission from the author. Additional information is available at http://www. geochemicalperspectivesletters.org/copyright-and-permissions.

Cite this letter as: Ozawa, K., Hirose, K., Kuwayama, Y., Takahashi, Y. (2021) The pressure-induced local structural change around tungsten in silicate glass. Geochem. Persp. Let. $18,6-10$

\section{References}

Bajgain, S., GHOSH, D.B., KaRKI, B.B. (2015) Structure and density of basaltic melts at mantle conditions from first-principles simulations. Nature Communications 6,8578

Cottrell, E., Walter, M.J., Walker, D. (2009) Metal-silicate partitioning of tungsten at high pressure and temperature: Implications for equilibrium core formation in Earth. Earth and Planetary Science Letters 281, 275-287.

de Grouchy, C.J.L., Sanloup, C., Cochain, B., Drewitt, J.W.E., Kono, Y., CRÉPISSON, C. (2017) Lutetium incorporation in magmas at depth: Changes in melt local environment and the influence on partitioning behaviour. Earth and Planetary Science Letters 464, 155-165.

Farges, F., Linnen, R.L., Brown, G.E. JR. (2006) Redox and speciation of tin in hydrous silicate glasses: A comparison with $\mathrm{Nb}, \mathrm{Ta}, \mathrm{Mo}$ and $\mathrm{W}$. The Canadian Mineralogist 44, 775.

Hin, R.C., Burnham, A.D., Gianolio, D., Walter, M.J., Elliot, T. (2019) Molybdenum isotope fractionation between $\mathrm{Mo}^{4+}$ and $\mathrm{Mo}^{6+}$ in silicate liquid and metallic Mo. Chemical Geology 504, 177-189.

Jennings, E.S., Jacobson, S.A., Rubie, D.C., Nakajima, Y., Vogel, A.K., RoseWeston, L.A., Frost, D.J. (2020) Metal-silicate partitioning of $W$ and Mo and the role of carbon in controlling their abundances in the bulk silicate earth. Geochimica et Cosmochimica Acta 293, 40-69.

Kashimabara, T., Takahashi, Y., Marcus, M.A., Uruga, T., Tanida, H., Terada, Y, Usu, A. (2013) Tungsten species in natural ferromanganese oxides related to its different behavior from molybdenum in oxic ocean. Geochimica et Cosmochimica Acta 106, 364-378.

Kashiwabara, T., Kubo, S., Tanaka, M., Senda, R., Izzuka, T., Tanimizu, M., TAKAHASHI, Y. (2017) Stable isotope fractionation of tungsten during adsorption on Fe and $\mathrm{Mn}$ (oxyhydr)oxides. Geochimica et Cosmochimica Acta 204, 52-67.

KeppleR, H., Rubie, D.C. (1993) Pressure-induced coordination changes of transition-metal ions in silicate melts. Science 364, 54-56.

Leroy, C., Sanloup, C., Bureau, H., Schmidt, B.C., Konopkova, Z., Raepsaet, C. (2018) Bonding of xenon to oxygen in magmas at depth. Earth and Planetary Science Letters 484, 103-110.

McDonough, W.F. (2014) Compositional model for the Earth's core. In: Holland, H.D., TureKian, K.K. (Eds.) Treatise on Geochemistry. Second Edition, Elsevier, Oxford, 559-577.

O'Neill, H.S.C., BerRY, A.J., EgGins, S.M. (2008) The solubility and oxidation state of tungsten in silicate melts: Implications for the comparative chemistry of $\mathrm{W}$ and Mo in planetary differentiation processes. Chemical Geology 255, 346-359.

PAuling, L. (1929) The principles determining the structure of complex ionic crystals. Journal of American Chemical Society 51, 1010-1026.

Prescher, C., PrakapenKa, V.B., Stefanski, J., Jahn, S., Skinner, L.B., Wang, Y. (2017) Beyond sixfold coordinated $\mathrm{Si}$ in $\mathrm{SiO}_{2}$ glass at ultrahigh pressures. Proceedings of the National Academy of Sciences 114, 10041-10046.

RAI, N., VAN WESTRENEN, W. (2014) Lunar core formation: New constraints from metal-silicate partitioning of siderophile elements. Earth and Planetary Science Letters 388, 343-352. 
Sanloup, C., van Westrenen, W., Dasgupta, R., Maynard-Casely, H., Perrillat, J. (2011) Compressibility change in iron-rich melt and implications for core formation models. Earth and Planetary Science Letters 306, 118-122.

Sanloup, C., Drewitt, J.W.E., Konopkova, Z., Dalladay-Simpson, P., Morton, D.M., Rai, N., van Westrenen, W., Morgenroth, W. (2013) Structural change in molten basalt at deep mantle conditions. Nature 503, 104-107.

SATO, T., FunAmori, N. (2010) High-pressure structural transformation of $\mathrm{SiO}_{2}$ glass up to 100 GPa. Physical Review B 82, 184102.

SHANNON, R. (1976) Revised effective ionic radii and systematic studies of interatomic distances in halides and chalcogenides. Acta Crystallographica Section A 32, 751-767.

SHIBAZAKI, Y., Kono, Y., FeI, Y. (2015) Microscopic structural change in a liquid Fe-C alloy of $~ 5$ GPa. Geophysical Research Letters 42, 13.

Siebert, J., CORGNe, A., RYERSON, F.J. (2011) Systematics of metal-silicate partitioning for many siderophile elements applied to Earth's core formation. Geochimica et Cosmochimica Acta 75, 1451-1489.

WADE, J., WoOD, B.J., TuFF, J. (2012) Metal-silicate partitioning of Mo and W at high pressures and temperatures: Evidence for late accretion of sulphur to the Earth. Geochimica et Cosmochimica Acta 85, 58-74.

Walter, M.J., Thibault, Y. (1995) Partitioning of tungsten and molybdenum between metallic liquid and silicate melt. Science 270, 1186-1189. 